\title{
Editorial: Topical Collection on the Lomonosov Mission
}

\author{
Yuri Y. Shprits ${ }^{1,2,3}$
}

Received: 5 June 2017 / Accepted: 20 June 2017 / Published online: 10 August 2017

(C) Springer Science+Business Media B.V. 2017

Observations of space processes provide a unique opportunity to study fundamental physical and astrophysical processes in settings that cannot be reproduced in laboratories on the ground. Observations in space can help us to better quantify and understand the space environment, and help to reveal fundamental physical processes that occur around us and in the universe at large. So far, the Lomonosov mission is the largest satellite mission led by a university in Russia and is also the first mission that was launched from the new launch facility Vostochniy. The Lomonosov satellite was put in a Sun-synchronous orbit on a Soyuz launch vehicle on April 28, 2016. The launch mass was $625 \mathrm{~kg}$, with a payload mass of $290 \mathrm{~kg}$. The mission is planned for 3 years.

The Lomonosov scientific experiments focus on a number of critical and unanswered problems in space sciences, many of which have garnered scientific attention for several decades. An international team comprised of scientists from Russia, the US, Mexico, Korea, Germany, Taiwan, Spain, and Denmark conduct scientific experiments on board of Lomonosov. The experiments are described in this topical collection.

Sadovnichii et al. give an overview of the mission and the scientific experiments on board of Lomonosov. Some of the central experiments focus on understanding the most energetic particles, including the BDRG-GRB monitor (Amelushkin et al.), SHOCK (Lipunov et al.), TUS (Panasyuk et al.), and UFFO (Park et al.; Jeong et al.).

The Lomonosov Mission

Edited by Yuri Shprits, Hans Bloemen and Jim Burch

Y.Y. Shprits

yshprits@gfz-potsdam.de

1 Helmholtz Centre Potsdam, GFZ German Research Centre for Geosciences, Telegrafenberg, 14473 Potsdam, Germany

2 Department of Geophysics, University of Potsdam, Am Neuen Palais, 10, 14469 Potsdam, Germany

3 Institute for Physics and Astronomy, UCLA Department of Earth, Planetary, and Space Sciences, Los Angeles, CA, USA 
Lomonosov has two experiments that focus on energetic particles in the Earth's Van Allen radiation belts: ELFIN (Shprits et al.) and DEPRON (Benghin et al.). Near-Earth space is now home to many operational satellites and the International Space Station, and it is important to study the effects of energetic particles on technology in space and on the health of astronauts. The IMISS-1 experiment addresses another problem related to the health of astronauts: gaze-stabilization delay under microgravity (Sadovnichii et al.).

The results of the instruments will be made available to the broader scientific community involving a number of scientists from around the world. 